\title{
A Clinical Audit of Adherence to Guidelines During Emergency Management of Pediatric Dengue Patients
}

\author{
Bharat Choudhary ${ }^{1}$ (D) $\cdot$ Manjesh Jayappa ${ }^{2} \cdot$ Priya Gupta $^{2} \cdot$ Kuldeep Singh $^{2} \cdot$ Yachana Choudhary $^{3}$
}

Received: 4 December 2020 / Accepted: 15 April 2021 / Published online: 28 April 2021

(C) Dr. K C Chaudhuri Foundation 2021

To the Editor: Dengue infection has emerged as an endemic in almost all states of India [1]. Children share a disproportionate burden in the prevalence and are especially vulnerable to complications $[2,3]$. Clinical audit serves as a reliable tool in gauging the effectiveness and performance of care provided as compared to standard of care [4]. We performed a clinical audit of pediatric dengue case management at our emergency department.

This was a retrospective record-based descriptive study conducted from September 2018 to January 2019. Data extraction was done by two clinicians independently. For each case, the accessors marked the compliance to the latest WHO protocol for assessment, diagnosis, treatment, and discharges with comments. During the study period, 408 children presented with complaints of fever, of whom, 160 were treated as dengue fever on clinical suspicion; however, $81 \%$ (131) children had laboratory evidence of dengue. We found $94 \%$ compliance in initial assessment, 95\% (67/71) documenting correct diagnosis; conversely, $3 \%$ children were overdiagnosed to a severe category and $2 \%$ of children were recorded to have a less severe category. Treatment compliance for initial fluid therapy was $85 \%$ (62/73), for colloids $75 \%$ (3/4), and for platelets transfusion was $100 \%$ (4/4). Colloid solution was not used for 1 patient when it was indicated. The common error identified was initiating a low fluid rate $(3 \mathrm{~mL} /$ $\mathrm{kg} / \mathrm{h}$ ) as compared to $5-7 \mathrm{~mL} / \mathrm{kg} / \mathrm{h}$ in children with warning signs; $93 \%$ of children were advised about fluid therapy and

Bharat Choudhary

drbharatpaeder@gmail.com

1 Department of Trauma and Emergency (Pediatrics), All India Institute of Medical Sciences, Jodhpur, Rajasthan 342005, India

2 Department of Pediatrics, All India Institute of Medical Sciences, Jodhpur, Rajasthan, India

3 Department of Community Medicine \& Family Medicine, All India Institute of Medical Sciences, Jodhpur, Rajasthan, India danger signs but none was advised about mosquito bite prevention strategies at discharge. Post-audit findings were communicated to all residents; triage sheet used at emergency was modified to include body weight and a dedicated discharge instruction sheet was prepared. This audit has given us opportunity to reflect upon our practice and adopt the necessary changes, which will further improve care of the children at our institute.

\section{Declarations}

Ethical Approval The study was approved by the ethics committee of AIIMS Jodhpur.

Conflict of Interest None.

\section{References}

1. Ganeshkumar P, Murhekar MV, Poornima V, et al. Dengue infection in India: a systematic review and meta-analysis. PLoS Negl Trop Dis. 2018;12(7):e0006618.

2. Verhagen LM, de Groot R. Dengue in children. J Infect. 2014;69(Suppl 1):S77-86.

3. Low GK-K, Ogston SA, Yong M-H, Gan S-C, Chee H-Y. Global dengue death before and after the new World Health Organization 2009 case classification: a systematic review and meta-regression analysis. Acta Trop. 2018;182:237-45.

4. Paton JY, Ranmal R, Dudley J, RCPCH Clinical Standards Committee. Clinical audit: still an important tool for improving healthcare. Arch Dis Child Educ Pract Ed. 2015;100:83-8.

Publisher's Note Springer Nature remains neutral with regard to jurisdictional claims in published maps and institutional affiliations. 